\title{
WGAViewer: Software for genomic annotation of whole genome association studies
}

\author{
Dongliang Ge, ${ }^{1,2,5}$ Kunlin Zhang, ${ }^{3}$ Anna C. Need, ${ }^{1}$ Olivier Martin, ${ }^{4}$ Jacques Fellay, ${ }^{1,2}$ \\ Thomas J. Urban, ${ }^{1,2}$ Amalio Telenti, ${ }^{2,3}$ and David B. Goldstein ${ }^{1,2,5}$ \\ ${ }^{1}$ Center for Population Genomics \& Pharmacogenetics, Institute for Genome Sciences \& Policy, Duke University, \\ Durham, North Carolina 27708, USA; ${ }^{2}$ Center for HIV/AIDS Vaccine Immunology, Duke University, \\ Durham, North Carolina 27708, USA; ${ }^{3}$ Institute of Microbiology, University Hospital, University of Lausanne, \\ 1011 Lausanne, Switzerland; ${ }^{4}$ Swiss Institute of Bioinformatics, 1015 Lausanne, Switzerland
}

\begin{abstract}
To meet the immediate need for a framework of post-whole genome association (WGA) annotation, we have developed WGAViewer, a suite of JAVA software tools that provides a user-friendly interface to automatically annotate, visualize, and interpret the set of $P$-values emerging from a WGA study. Most valuably, it can be used to highlight possible functional mechanisms in an automatic manner, for example, by directly or indirectly implicating a polymorphism with an apparent link to gene expression, and help to generate hypotheses concerning the possible biological bases of observed associations. The easily interpretable diagrams can then be used to identify the associations that seem most likely to be biologically relevant, and to select genomic regions that may need to be resequenced in a search for candidate causal variants. In this report, we used our recently completed study on host control of HIV-1 viral load during the asymptomatic set point period as an illustration for the heuristic annotation of this software and its contributive role in a successful WGA project.
\end{abstract}

[Supplemental material is available online at www.genome.org. WGAViewer is available at http://www.genome. duke.edu / centers / pg2/ downloads / wgaviewer.php.]

Dramatic advances in genotyping technologies have allowed the development of affordable products that simultaneously genotype a genome-wide set of polymorphisms that are known to represent most of the common genetic variants in specific human population groups. The relatively consistent properties of tagging single nucleotide polymorphisms (SNPs) across different human populations imply that such SNP sets may be sufficient for use in association studies for most populations (Need and Goldstein 2006). Because of these developments, the use of HapMap data (The International HapMap Consortium 2005) and other genome resources has shifted from upstream SNP-selection tasks to the downstream tasks of interpreting the observed genotype-phenotype associations (Telenti and Goldstein 2006). Ideally, these resources should be used not only to help distinguish real associations from false-positive ones, but also to help generate hypotheses concerning the possible biological basis of observed associations.

To address these needs it is necessary to develop an annotation environment that will allow automated interpretation of a large set of $P$-values in the context of known genomic features and also in the context of other studies of similar phenotypes. Such methods would allow investigators to consider and interpret the full set of $P$-values resulting from an association study in an automatic and convenient manner. Most importantly, it would highlight possible mechanisms, for example, by directly or indirectly implicating a polymorphism with an apparent link to gene expression, splicing, noncoding RNAs, and other possibilities suggesting specific functional tests.

Here, we introduce WGAViewer, a suite of JAVA software tools that provides a user-friendly interface to annotate, visual-

\footnotetext{
${ }^{5}$ Corresponding authors.

E-mail d.ge@duke.edu; fax (919) 668-6787.

E-mail d.goldstein@duke.edu; fax (919) 668-6787.

Article published online before print. Article and publication date are at http:// www.genome.org/cgi/doi/10.1101/gr.071571.107.
}

ize, and help interpret the set of $P$-values emerging from a whole genome association (WGA) study. The program connects to the latest online genomic databases to annotate the SNPs and their associated $P$-values in the context of predicted gene structure and SNP function (Hubbard et al. 2007), association with gene expression (Stranger et al. 2007), evidence of recent selection (Voight et al. 2006), and concurrent evidence from multiple association studies. HapMap data (The International HapMap Consortium 2005) is used to identify non-genotyped polymorphisms that associate with the phenotype of interest through linkage disequilibrium (LD) with genotyped variants. The easily interpretable diagrams can be used to identify the associations that seem most likely to be biologically relevant, and to select genomic regions that may need to be resequenced in a search for candidate causal variants. Finally, we chose to use the term "whole genome" instead of the more common (and currently more accurate "genome-wide") because eventually WGAViewer will be developed to incorporate complete resequencing data.

\section{Results}

We have developed WGAViewer, a stand-alone JAVA software package. The current version (1.25) offers six classes of annotation: First, the genome overview and chromosomal views display the distribution of $P$-values across the entire genome or across individual chromosomes, allowing visualization of the top hits and point-and-click selection of regions to amplify for more detailed inspection. Second, the gene context view aligns the WGA results with the latest genome build (Hubbard et al. 2007) and displays the genotyped SNPs against gene maps that include known transcripts along with gene structure (Hubbard et al. 2007), LD pattern (The International HapMap Consortium 2005), and evidence of recent selection (Voight et al. 2006). Further information for genes, transcripts, exons, and SNPs is available via 
hyperlink. This annotation also displays concurrent evidence from multiple databases for convenient comparison. Third, the individual SNP annotation annotates the associated SNP with a graphical representation of its LD score with all HapMap SNPs in a specified surrounding region (The International HapMap Consortium 2005), so that non-genotyped associated SNPs in surrounding genes can be easily identified. This class of annotation also tests whether associated polymorphisms show any association with the expression (in immortalized B-lymphocytes) of the closest (or an alternatively specified) gene currently represented in the GENEVAR database (Stranger et al. 2007). This feature will be expanded as additional data become available, for example, to include data on both expression in other tissue types and alternative splicing. Displayed alongside this plot is additional SNPrelated information, such as ancestral allele, concurrent evidence from multiple databases, SNP function (synonymous, nonsynonymous, splice-site, etc.), validation status, and other identifiers. Fourth, the gene/SNP finding annotation provides a convenient way to locate and annotate candidate genes/SNPs of specific interest in a WGA project, and to align with the physical coordinates from the latest genome build. Moreover, this annotation enables a search for specific SNPs, and if they are not present in a WGA project, it then displays the results for the SNPs that are associated at a user-specified level (their "LD proxies"). These functions make a handy and reliable comparison with existing reports, for example, the reports from previous candidate gene studies. When specific SNPs of interest have not been genotyped in a WGA project, these annotations for searching $L D$ proxies are especially convenient and valuable. Fifth, concurrent evidence from multiple databases allows the user to load multiple databases simultaneously, with one of them as the "core" database to be considered. The supporting databases could include replication studies, projects with related phenotypes, other publicly available projects, even studies with different marker sets or different phenotypes. These data sets can then be listed and plotted alongside the core database as concurrent evidence. WGAViewer provides a convenient way to select, sort, and annotate the SNPs with evidence at specified significance level across all the core and reference databases. Finally, user-customized supporting/QC databases can be loaded by WGAViewer to facilitate the interpretation of the WGA results, including important information such as HWE $P$-values, effect size, effect direction, QC scores, or other user-customized data.

As an illustration of the heuristic annotation of WGAViewer and its contribution to a real WGA project, we use a recently completed study on host control of human immunodeficiency virus-1 (HIV-1) viral load during the asymptomatic set point period (Fellay et al. 2007). A screenshot from WGAViewer (Fig. 1) shows the individual SNP annotation results for rs9264942 on chromosome 6 spanning from 31,182 Kbp to 31,582 Kbp. First, an overview of chromosome 6 shows two genome-wide significant SNPs (plotted in red points). The further annotation then shows that one of the two hits, rs9264942, is located in the 5' region of the $H L A-C$ (major histocompatibility complex, class I, C) gene and is $\sim 35 \mathrm{~kb}$ away from the transcription initiation site. Most strikingly, WGAViewer explicitly shows that this SNP shows a high correlation with the expression levels of $H L A-C$, which is the closest gene to this SNP. These annotations immediately lead to a hypothesis that rs9264942 may function through controlling $H L A-C$ expression level and thus point to a most promising direction for functional investigations. However, it should also be noted that in the case of a highly variable gene like $H L A-C$, apparent expression effects could result from either an association with expression or an association with variant sites affecting probe binding (T. Urban, W. Yoon, K.V. Shianna, J. Fellay, D. Ge, B.H. Haynes, A.J. McMichael, M. Carrington, A. Telenti, and D.B. Goldstein, unpubl.). Furthermore, these annotations also enable the convenient identification of SNPs in LD with rs9264942, which are associated with $H L A$-C expression level and HIV-1 viral load, for example, rs2249742 $\left(r^{2}=0.74,1 \mathrm{~kb}\right.$ upstream). Finally, in addition to the individual result as shown in Figure 1, this class of annotations can be sequentially performed for a number of ranked SNPs at a specified cutoff.

\section{Discussion}

In this report we introduce WGAViewer, a JAVA software tool that provides a user-friendly interface to annotate and help interpret the set of $P$-values emerging from a whole genome association (WGA) study. Using the six classes of annotation that are provided by this software, different pipelines for annotation can be employed to interpret the results from a WGA project (see Supplemental material, WGAViewer User's Guide). Most importantly, this annotation environment provides a convenient and heuristically accessible summary of information that is relevant to the interpretation of association results. In our own experience, carrying out simple steps such as asking whether an associated polymorphism is in linkage disequilibrium with a functional polymorphism or whether it associates with the expression of nearby genes can take an unreasonably long time to reconcile necessary databases and make the comparisons. For example, when a specific polymorphism is implicated in an association study, an obvious and important immediate question is whether the polymorphism associates with expression levels of nearby genes. One data set available for performing this is the GENEVAR data set (Stranger et al. 2007), but extracting the necessary data from GENEVAR to perform the test is laborious. Specifically, it is necessary to first search for the closest gene to target SNPs using the latest genome build (for example, using the Ensembl database). Secondly, one has to search for the probes for this gene in the GENEVAR database and retrieve the individual normalized expression data in each HapMap cell line in the appropriate population. Thirdly, one has to search the HapMap database to retrieve the SNP genotype for each individual. And finally, one has to merge these data from disparate data sources and perform an association test. In our experience, these steps can take $75 \mathrm{~min}$ on average. WGAViewer, however, dramatically expedites these classes of interpretations in an automated manner and presents results in heuristic graphs. Using this software, it is extremely convenient to search for candidate genes, SNPs, and their LD proxies, requiring no need for input of genome coordinates or other information. The work session, along with downloaded annotation and interactive features, can be saved and reopened at a later date.

As such, this software tool can be viewed primarily as expediting the work that would always follow the discovery of strong associations with a phenotype of interest. Most of the features to be found in WGAViewer emerge directly from real studies in the lab where we asked specific questions for specific discoveries and found that the process would substantially benefit from automation (see Supplemental Table 1).

In addition to these expediting annotations, there is, however, one class of annotation that goes beyond convenient summaries of work that could be carried out manually and individu- 


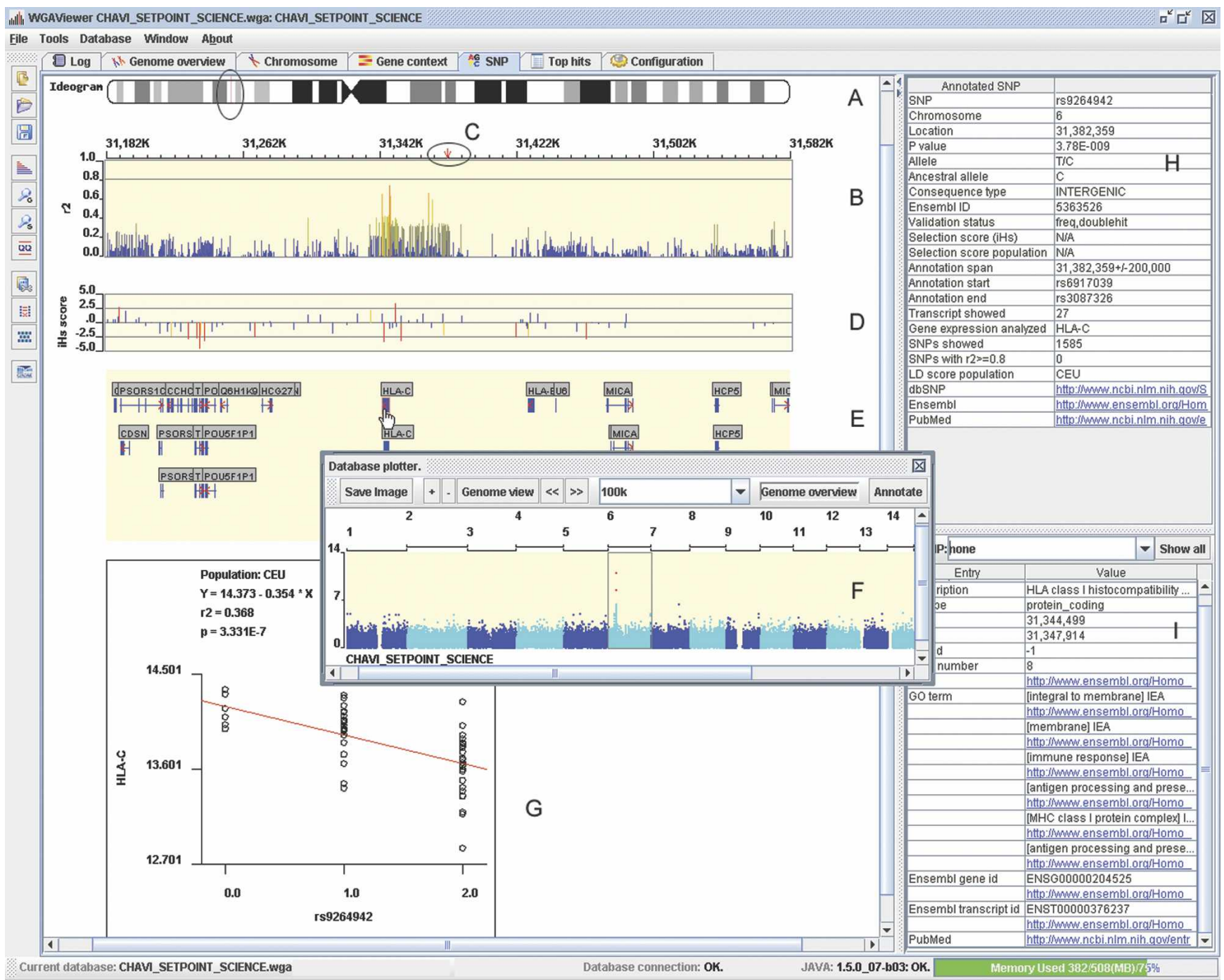

Figure 1. A screenshot of the results of WGAViewer individual SNP annotation for rs9264942 (Fellay et al. 2007). (A) Ideogram depicting chromosome and annotated region (circled red line). (B) Pairwise LD values $\left(r^{2}\right)$ between the associating SNP, rs9264942 (position indicated by circled red arrow), and all other HapMap SNPs in the region (SNP list from HapMap release 22, build 36; genome coordinates based on Ensembl release 46_36h, build 36; [red] $r^{2} \geq 0.8$, [yellow] $0.5 \leq r^{2}<0.8$, [gray] $0.3 \leq r^{2}<0.5$, [blue] $0.2 \leq r^{2}<0.3$, [dark gray] missing data). (C) Position of annotated SNP, rs9264942 (genome coordinate based on Ensembl release 46_36h, build 36). (D) Recent selection scores (Voight et al. 2006) for SNPs plotted in $B$ where available. (Red) liHsl $\geq 2.5$, (yellow) $2.0 \leq \mid \mathrm{iHsl}<2.5$, (blue) liHsl $<2.0$. (E) Gene diagram with introns (horizontal blue lines) and exons (vertical blue lines) depicted. Detailed information for the highlighted gene (HLA-C, with a hand cursor) is shown in a dynamic data sheet (part I). ( $F$ ) Genome overview of the WGA results. Two genome-wide significant hits (rs2395029 and rs9264942) are plotted (red points). (G) Association test between rs9264942 genotypes and HLA-C expression levels (Stranger et al. 2005), showing highly significant association. It should also be noted that in the case of a highly variable gene like $H L A-C$, apparent expression effects could result from either an association with expression or an association with variant sites affecting probe binding (T. Urban, W. Yoon, K.V. Shianna, J. Fellay, D. Ge, B.H. Haynes, A.J. McMichael, M. Carrington, A. Telenti, and D.B. Goldstein, unpubl.). (H) Data sheet summarizing important information for the annotated SNP, rs9264942. (I) Dynamic data sheet summarizing important information for items (HLA-C transcript ENST00000376237) highlighted in parts B-E. For detailed descriptions, see Supplemental material: WGAViewer User's Guide Chapter 3.3: Annotation for top hits.

ally. In our own experience, the manual annotations are not feasible beyond the few top discoveries. What happens with a polymorphism that achieves a $P$-value of only $10^{-5}$, but which is itself in strong LD with an ungenotyped SNP that is thought to have definite functional consequence? This SNP would warrant special consideration, but could be missed in most manual settings. We have established a comprehensive annotation (1-5 min per SNP) meant to pluck out this kind of suggestive associations, and this is one of the few features of WGAViewer that aims toward real automated consideration of the full sets of results, as opposed to only expediting and summarizing analyses. This function features a serial annotation for a set of SNPs ranked by their $P$-values. In addition to the gene context, LD extension, and expression annotation routines for each SNP, this process will also check and filter the functions for both the original genotyped SNPs and their LD proxies. Once the annotation is done, the interactive filtering and other annotation features can be saved for convenient later use.

The rapidly increasing number of genome scans using identical SNP sets allows assessment of common genetic determinants across different diseases and phenotypes. In order to facilitate searches for polymorphisms that impact multiple pheno-

\section{Genome Research}

www.genome.org 
types, we have incorporated features for comparing the evidence across databases, including between original findings and replication cohorts, between studies in various populations, between original findings and related phenotypes, or between original findings and results from different studies. For these purposes, WGAViewer has been designed to read and annotate multiple databases and to identify all SNPs that show a user-specified degree of association in two or more studies. WGAViewer provides a free and convenient platform to access and annotate all the WGA databases hosted by and released from the Duke Institute for Genome Sciences \& Policy, powered by the Mart for IGSP Data from Association Studies (an instance of BioMart; Kasprzyk et al. 2004). In addition to this, WGAViewer also provides a convenient interface that allows a user to load and list different kinds of supporting information that may facilitate the interpretation.

WGAViewer is an ongoing project under continuous development. Our future development plans currently include: transcription factor binding sites, miRNA sites, comparative genetics, and splicing regulators. We welcome suggestions and collaborations.

We view the publicly available WGAViewer tool as a first step toward facilitating the interpretation of genome-wide association studies in the context of the rapidly evolving knowledge of the human genome and the disparate databases that house that knowledge.

\section{Methods}

\section{Annotation implementation}

WGAViewer is a stand-alone software developed using the JAVA language by Sun Microsystems (http://java.sun.com/) and an integrated development environment (IDE) NetBeans (http:// www.netbeans.org/). WGAViewer was compiled and should be used in a computer system with a JAVA environment with version 1.5.0_07-b03 or later. The current version has 186 JAVA classes and is released in one executable JAR file. Microsoft Windows users have an option to launch the program through a wrapper program: WGAViewer.exe, while other users can start the program through a shell program, WGAViewer.sh. We detail the scheme of the WGAViewer annotation methods in Supplemental Figure 1.

\section{Alignments between genome builds}

Instead of using a fixed genome build version as the genomic coordinate source, we chose to always apply the latest genome build from Ensembl at the time of each annotation, and map every coordinate from other sources to this dynamic core database. This dynamic procedure helps to interpret the WGA results using the most updated transcripts and SNP coordinates, and avoids discrepancy between different major builds used in different sources (for example, build 36 and build 35), or even between different subversions (for example, build 36: Ensembl version $46.36 \mathrm{~h}$ and 43.36e). This procedure is implemented in all the annotation routines, most importantly, in the gene and SNP searching functions. We noticed that sometimes these discrepancies could be up to $\sim 200 \mathrm{~kb}$, which could potentially lead to very different gene/SNP contexts and hence result in different interpretations and hypotheses. Therefore, instead of directly using genome coordinates from diverse sources, we have treated the genetic markers as anchors and always used a fast hashtablematching to map these anchors into the uniform Ensembl build version. This uniform build version, together with the version from other sources, is stored in the annotated project file and can be referred to in the later interpretation.

\section{Testing}

We have used and tested WGAViewer on typical Windows- and Linux-based PCs (2-GHz processor, 2 GB of memory). A brief annotation for ranked hits without graphical features can take $0.5-1.0$ sec per SNP depending on Web connection speed. A comprehensive annotation with a spanning region $\sim 200 \mathrm{~kb}$ can take 1-5 min per SNP. For a typical WGA result set with $\sim 550,000$ SNPs, WGAViewer requires at least $512 \mathrm{Mb}$ of memory to load and store the annotation results, with a tool for monitoring memory usage, and will alert the user when the system is about to run out of memory. WGAViewer does require that the user computer system has the ability to establish a standard MySQL database connection, which is through the most common MySQL port 3306 and 5306. In cases where such connections cannot be directly established, WGAViewer provides a substitutive proxy connection through the servers hosted in the Duke Institute for Genome Sciences \& Policy.

WGAViewer has been applied in a number of projects (Cavalleri et al. 2007; Fellay et al. 2007). A data set based on host control of HIV-1 viral load has been included in the released package as an example.

\section{Acknowledgments}

We thank Darin London, Robert J. Wagner, and Mark R. DeLong from the Duke Institute for Genome Sciences \& Policy for their work on setting up the query engine of the Mart for IGSP Data from Association Studies (MIDAS), provided by the Biomart Data Integration System (http://www.biomart.org/). WGAViewer is supported by the Center for HIV-AIDS Vaccine Immunology (CHAVI, http://www.chavi.org/) and the Duke Institute for Genome Sciences \& Policy (IGSP, http://www.genome.duke.edu/).

\section{References}

Cavalleri, G.L., Weale, M.E., Shianna, K.V., Singh, R., Lynch, J.M., Grinton, B., Szoeke, C., Murphy, K., Kinirons, P., O'Rourke, D., et al. 2007. Multicentre search for genetic susceptibility loci in sporadic epilepsy syndrome and seizure types: A case-control study. Lancet Neurol. 6: 970-980.

Fellay, J., Shianna, K.V., Ge, D., Colombo, S., Ledergerber, B., Weale, M., Zhang, K., Gumbs, C., Castagna, A., Cossarizza, A., et al. 2007. A whole-genome association study of major determinants for host control of HIV-1. Science 317: 944-947.

Hubbard, T.J., Aken, B.L., Beal, K., Ballester, B., Caccamo, M., Chen, Y., Clarke, L., Coates, G., Cunningham, F., Cutts, T., et al. 2007. Ensembl 2007. Nucleic Acids Res. 35: D610-D617.

The International HapMap Consortium. 2005. A haplotype map of the human genome. Nature 437: 1299-1320.

Kasprzyk, A., Keefe, D., Smedley, D., London, D., Spooner, W., Melsopp, C., Hammond, M., Rocca-Serra, P., Cox, T., and Birney, E. 2004. EnsMart: A generic system for fast and flexible access to biological data. Genome Res. 14: 160-169.

Need, A.C. and Goldstein, D.B. 2006. Genome-wide tagging for everyone. Nat. Genet. 38: 1227-1228.

Stranger, B.E., Forrest, M.S., Clark, A.G., Minichiello, M.J., Deutsch, S., Lyle, R., Hunt, S., Kahl, B., Antonarakis, S.E., Tavare, S., et al. 2005 Genome-wide associations of gene expression variation in humans. PLoS Genet. 1: e78. doi: 10.1371/journal.pgen.0010078.

Stranger, B.E., Forrest, M.S., Dunning, M., Ingle, C.E., Beazley, C., Thorne, N., Redon, R., Bird, C.P., de Grassi, A., Lee, C., et al. 2007. Relative impact of nucleotide and copy number variation on gene expression phenotypes. Science 315: 848-853.

Telenti, A. and Goldstein, D.B. 2006. Genomics meets HIV-1. Nat. Rev. Microbiol. 4: 865-873.

Voight, B.F., Kudaravalli, S., Wen, X., and Pritchard, J.K. 2006. A map of recent positive selection in the human genome. PLoS Biol. 4: e72. doi: 10.1371/journal.pbio.0040072.

Received September 25, 2007; accepted in revised form January 25, 2008. 


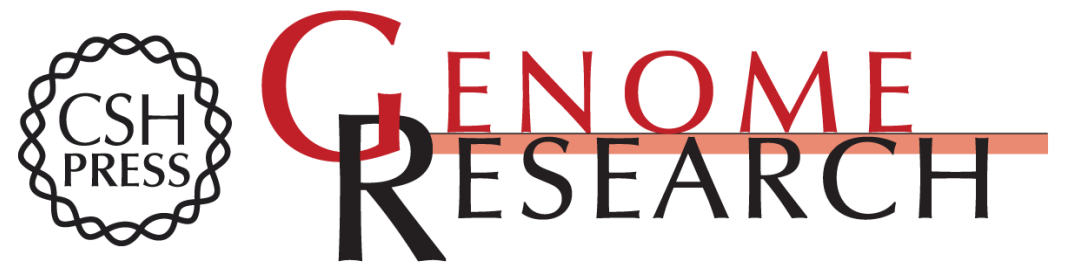

\section{WGAViewer: Software for genomic annotation of whole genome association studies}

Dongliang Ge, Kunlin Zhang, Anna C. Need, et al.

Genome Res. 2008 18: 640-643

Access the most recent version at doi:10.1101/gr.071571.107

Supplemental Material

References

License

Email Alerting Service
http://genome.cshlp.org/content/suppl/2008/03/04/gr.071571.107.DC1

This article cites 10 articles, 3 of which can be accessed free at: http://genome.cshlp.org/content/18/4/640.full.html\#ref-list-1

Receive free email alerts when new articles cite this article - sign up in the box at the top right corner of the article or click here.

\section{Affordable, Accurate Sequencing.}

